\title{
Smartphone Drum: Gesture-based Digital Musical Instruments Application for Deaf and Hard of Hearing People
}

\author{
Ryo Iijima \\ ryoiijima@digitalnature.slis.tsukuba.ac.jp \\ University of Tsukuba \\ Tsukuba, Japan \\ Sayan Sarcar \\ mailtosayan@gmail.com \\ University of Tsukuba, Japan \\ Birmingham City University, UK
}

\author{
Akihisa Shitara \\ theta-akihisa@digitalnature.slis.tsukuba.ac.jp \\ University of Tsukuba \\ Tsukuba, Japan \\ Yoichi Ochiai \\ wizard@digitalnature.slis.tsukuba.ac.jp \\ University of Tsukuba \\ Tsukuba, Japan
}

\begin{abstract}
Smartphone applications that allow users to enjoy playing musical instruments have emerged, opening up numerous related opportunities. However, it is difficult for deaf and hard of hearing (DHH) people to use these apps because of limited access to auditory information. When using real instruments, DHH people typically feel the music from the vibrations transmitted by the instruments or the movements of the body, which is not possible when playing with these apps. We introduce "smartphone drum," a smartphone application that presents a drum-like vibrotactile sensation when the user makes a drumming motion in the air with their smartphone like a drumstick. We implemented an early prototype and received feedback from six DHH participants. We discuss the technical implementation and the future of new instruments of vibration.
\end{abstract}

\section{KEYWORDS}

Music, Game, User study, Deaf, Hard of hearing

\section{ACM Reference Format:}

Ryo Iijima, Akihisa Shitara, Sayan Sarcar, and Yoichi Ochiai. 2021. Smartphone Drum: Gesture-based Digital Musical Instruments Application for Deaf and Hard of Hearing People. In . ACM, New York, NY, USA, 2 pages.

\section{INTRODUCTION AND RELATED WORK}

Playing music can strengthen our creativity [1]. In an effort to make these benefits available to more diverse groups of people, accessible digital musical instruments (ADMI) are gaining interest in the field of computer music [3]. Prior research has aided DHH people in playing musical instruments through sensory substitution. However, it has been suggested that cost is an obstacle to both creation and utilization of accessibility technologies because products and services that require specialized devices can be difficult to afford by those with limited income.

Considering this, we aimed to create a musical instrument that does not rely on sound, using widespread smartphones to realize accessible instruments. To achieve this, we introduce the "smartphone drum," a smartphone application that presents a drum-like vibrotactile sensation when a user makes a drumming motion in the air using their smartphone as a drumstick.

\section{(c) 2021}

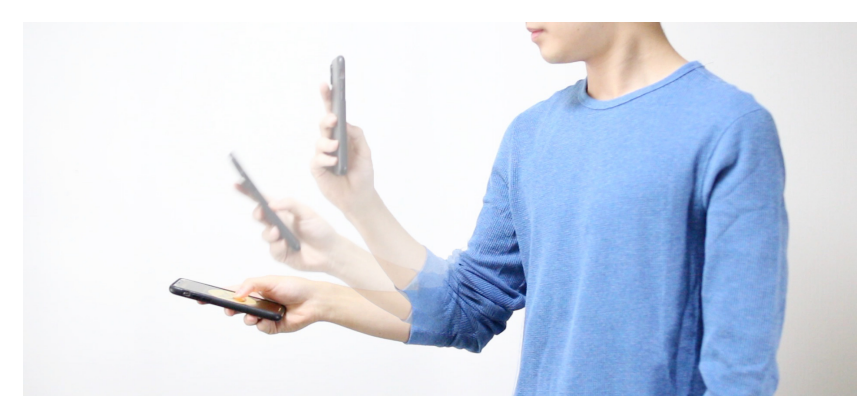

Figure 1: A User playing the smartphone drum. It detects the drumming motion and presents a drum-like vibrotactile sensation.

\section{INITIAL PROTOTYPE}

\subsection{Instrument Design}

We first discussed with our co-author, Shitara, who has profound hearing loss, regarding the considerations to be made for $\mathrm{DHH}$ people to enjoy musical instruments on their smartphones. We identified the following requirements:

- Requires less musical knowledge. Some DHH people may have little or no musical experience. Therefore, the design should be enjoyable for both novices and experts.

- Model a musical instrument whose vibrations are easily perceived. Among real instruments, percussion instruments have more recognizable vibrations than pianos or guitars. Such instruments might be better accepted by DHH people [2].

- Present rich feedback. The sound of acoustic instruments differs depending on subtle differences in the performer's movements. Rich feedback should be presented through vibrations to make the system work as a musical instrument and to convey the nuances.

With the above requirements in mind, we decided to design an instrument modeled after a drum. This is because drums can be enjoyed even without any prior experience or knowledge, and the vibrations transmitted from the drumsticks to the fingertips can be easily perceived. Furthermore, the beating motion is simple and easy to recognize, making it easy to follow the rhythm with the whole body. 
We created a prototype application that uses the smartphone as a drumstick, and when the user makes a drumming motion in the air, the smartphone produces a vibration as if the user has hit an actural drum. The envelope of the vibration changes depending on the strength of the beating motion. We prepared three types of drums: hi-hat, snare, and bass; therefore, the characteristics of the vibration would vary depending on the type of drum.

\subsection{Implementation}

The prototype was developed as an iOS application. Core Haptics, a framework for providing customized haptic feedback, is used to present a vibrotactile sensation.

Detection of Drumming Motion. The drumming motion is detected using the values obtained from the built-in accelerometer and gyroscope. When the user presses and holds a finger somewhere on the screen, the system starts capturing the values of the motion sensors at $100 \mathrm{~Hz}$ until the user takes the finger off the screen. When all of the following conditions were met, a drumming motion was considered to have been performed.

- (current z-axis acceleration $)<1$

- (previous $x$-axis gyro) $<0$

- (current $\mathrm{x}$-axis gyro) $<$ (previous $\mathrm{x}$-axis gyro)

Generating Haptic Pattern. When a drumming motion is detected, the attitude of the device at that time is obtained, and the pitch value is used to determine the drum (among hi-hat, snare, and bass) that was hit. A vibration pattern is then generated using the drum-type and acceleration values as inputs. Core haptics provides two types of haptic patterns: transient (short and fixed duration) and continuous (customizable duration). In addition, intensity and sharpness (i.e., frequency) can be finely controlled. This gives us fine control over the intensity and frequency of drum vibrations, as well as their time variations.

\section{USER FEEDBACK}

\subsection{Experiment}

Participants. We recruited six DHH participants (three females and three males) through email, social media, and snowball sampling. Participants were 24 years old on average $(\mathrm{SD}=4.86$, range $=19$ 33). Participants were paid $¥ 430$ for the 30 -minute study period.

Procedure. We shared our iOS app with the participants to complete the entire study remotely. In the first half of the study, we started with a background questionnaire, explained how to use the application, and then let the participants play freely for a minimum of 90 seconds. In the second half, semi-structured interviews were conducted to ask them about their experiences using the app. The purpose of the semi-structured interview was to receive feedback on a possible direction for design and functionality.

\subsection{Result}

The results of the semi-structured interviews were open-coded and organized into the following three main themes:
Past musical experience. All participants had played the drums before, but it was only P3 who had heard the sound. P3 was interested in knowing how close the feedback was to the real feeling of the sound, rather than the vibration of the drum . Meanwhile, P6 said "the vibration from the app is more amusing than that from actual drums but is lesser." Users who have heard the actual sound seem to prefer feedback that is closer to the sound of actual drums, whereas users who have never heard the sound seem to prefer feedback similar to the vibrations transmitted by actual drums.

Variation of Musical Instruments. After experiencing our app, four participants said they would like to play other instruments with vibrations. Suggestions include other types of drums, such as Japanese drums (P1), shakers like maracas (P2), string instruments like viola, castanets, and tambourines (P3), and guitar (P4). There were also several suggestions for performing gestures. For example, P6 said it would be fun to have more variations of the drumming motions, such as swinging down with a wrist twisting motion.

Cooperative Play. P1 and P2 said that they wanted to play an ensemble with several people, with one person in charge of one scale. P2 reflected on his experiences of playing handbell ensembles at school, and said that he wanted to play with his friends.

\section{CONCLUSION AND FUTURE WORK}

We designed the vibration patterns empirically, which may have caused discrepancies between a smartphone drum and acoustic drums, specifically for those who have heard the sound of acoustic drums. Exploring the design space of vibration patterns that are comfortable for users will be the subject of a future study.

The primary objective behind this research was to assess the acceptance of the concept of a vibration-based musical instrument smartphone-app by DHH users and to understand their needs and capabilities. Therefore, it remains as a future task to investigate the effects of the nature and delay of vibrations on user experience.

Based on feedback from users, we would like to consider creating instruments other than drums, as well as creating a system for multiple users to play together.

\section{ACKNOWLEDGMENTS}

This work was supported by JST CREST Grant Number JPMJCR19F2, Japan.

\section{REFERENCES}

[1] Birgitta Cappelen and Anders-Petter Andersson. 2012. Musicking Tangibles for Empowerment. In Computers Helping People with Special Needs, Klaus Miesenberger, Arthur Karshmer, Petr Penaz, and Wolfgang Zagler (Eds.). Springer Berlin Heidelberg, Berlin, Heidelberg, 254-261. https://doi.org/10.1007/978-3-642-31522-0 38

[2] Rumi Hiraga and Nobuko Kato. 2006. Understanding Emotion through Multimedia: Comparison between Hearing-Impaired People and People with Hearing Abilities. In Proceedings of the 8th International ACM SIGACCESS Conference on Computers and Accessibility (Portland, Oregon, USA) (Assets '06). Association for Computing Machinery, New York, NY, USA, 141-148. https://doi.org/10.1145/1168987.1169012

[3] Alon Ilsar and Gail Kenning. 2020. Inclusive Improvisation through Sound and Movement Mapping: From DMI to ADMI (ASSETS '20). Association for Computing Machinery, New York, NY, USA, Article 49, 8 pages. https://doi.org/10.1145/ 3373625.3416988 